\title{
Statistical approach to tunneling time in attosecond experiments
}

\author{
Durmuş Demir ${ }^{\mathrm{a}}$, Tuğrul Güner ${ }^{\mathrm{b}, *}$ \\ a Department of Physics, Izmir Institute of Technology, IZTECH, TR35430, Izzmir, Turkey \\ b Department of Materials Science and Engineering, İzmir Institute of Technology, IZTECH, TR35430, Izmir, \\ Turkey
}

\section{A R T I C L E I N F O}

\section{Article history:}

Received 12 April 2017

Accepted 22 September 2017

Available online 28 September 2017

\section{Keywords:}

Quantum tunneling

Tunneling time

Attosecond science

Foundations of quantum mechanics

Entropy

\begin{abstract}
A B S T R A C T
Tunneling, transport of particles through classically forbidden regions, is a pure quantum phenomenon. It governs numerous phenomena ranging from single-molecule electronics to donoracceptor transition reactions. The main problem is the absence of a universal method to compute tunneling time. This problem has been attacked in various ways in the literature. Here, in the present work, we show that a statistical approach to the problem, motivated by the imaginary nature of time in the forbidden regions, lead to a novel tunneling time formula which is real and subluminal (in contrast to various known time definitions implying superluminal tunneling). In addition to this, we show explicitly that the entropic time formula is in good agreement with the tunneling time measurements in laser-driven He ionization. Moreover, it sets an accurate range for long-range electron transfer reactions. The entropic time formula is general enough to extend to the photon and phonon tunneling phenomena.
\end{abstract}

(c) 2017 Elsevier Inc. All rights reserved.

\section{Introduction}

Tunneling, transport of subatomic particles through the regions of space forbidden to classical motion, is a pure quantum phenomenon. Its physical relevance was first established by Gamow in his analysis of the $\alpha$-decay [1-3]. The tunnel diode [4,5] of Esaki was its first technological application. Undoubtedly, scanning tunneling microscope (STM) [6] of Binning and Rohrer started a new pace

\footnotetext{
* Corresponding author.

E-mail address: tugrulguner@iyte.edu.tr (T. Güner).
} 
in scientific and technological advancements. Today, for example, it is known that electron transfer reactions involve tunneling as the underlying mechanism. It governs acceptor-donor transition processes so that charge separation by electron transfer reaction takes place at photosynthetic reaction centers [7] after excited electrons are transferred by antenna pigments in consequence of the coherent electron energy transfer [8] during the photosynthesis. Tunneling is a ubiquitous mechanism that underlies numerous physical [9], chemical [10], biological [11] and technological phenomena [12].

Tunneling time, the time elapsed during the tunneling process, is crucial for determining reaction speeds of tunneling-enabled rare processes ranging from high-speed electronic devices to nuclear fusion. Moreover, it also plays an important role while determining the electron transfer reaction rates based on the interaction of electron with the corresponding vibrational mode of the molecule [13]. Recently, transition from region where Born-Oppenheimer approximation holds, to region where it breaks down is also investigated in terms of tunneling time [14]. In fact, with the advent of strong laser ionization experiments [15-19], it is becoming possible to measure the tunneling time [20,21] where certain metrological problems $[22,23]$ with the detection of the tunneling particle are shown to be surmountable [24,25]. Strong laser fields enable electrons to tunnel out of atoms, where the potential barrier formed forms a testbed for models of tunneling time [26,27].

Tunneling time depends on what kinetic theory is set forth for tunneling process, and therefore, the literature consists of various time definitions [28-31]. They include traversal time through modulated barriers [32-35], spin precession time [36-38], flux-flux correlation duration [39], phase stationary time [40-42], and Feynman path integral (FPI) averaging of the classical time [43-45]. Some of them are complex, some are difficult to associate with tunneling and some suffer from superluminality. Interestingly, contrary to their raison d'etre, all these tunneling times utilize a sort of time operator since they involve derivatives with respect to energy or potential. This is not the case for FPI averaging yet the resulting time is still controversial because classical trajectories live in imaginary time and their probability amplitudes interfere [46-50]. At present, the problem with these and other tunneling time definitions is that they seem incapable of explaining the experimental data as was comparatively analyzed and experimented in [51]. In view of the growing scientific and technological needs, however, it is necessary to have a working model that can reliably estimate the tunneling time for a given potential barrier.

The present work reports on a novel formulation of the tunneling time. The formulation, based on a statistical description of the evanescing particle in the classically forbidden region, gives a tunneling time which shows good agreement with the experimental data compared to all the widely-used time definitions. The essence of the formulation is that, in the classically forbidden region time flows in imaginary direction, and correspondence between imaginary time in quantum mechanics and temperature in statistical mechanics enables a statistical formulation for tunneling. The resulting thermal energy, through the uncertainty principle, sets a generalized time interval depending on the transmission amplitude. Next, this model is applied numerically to the laser-driven He ionization covering recent experimental data [51] and to the electron transfer reactions. It is found that entropic tunneling time is in good agreement with the experimental data for the former, and sets the validity range of electron transitions in the long range electron transfer reactions, for the latter.

The energy-time uncertainty, which we utilize for the thermal energy of the tunneling particle, has been utilized in a different tunneling time study [52]. In that related work, it is assumed that exchange between the kinetic energy of the electron and the potential energy describing the tunneling region leads to an uncertainty in the total energy of the electron. It is then taken that the uncertainty in this total energy is proportional to the potential energy at the exit point of the electron $\Delta E \propto\left|V_{\text {exit }}\right|$. The model potentials used in [52], which are based on effective nuclear charge models described already in Section 4.1, are also used in this study to compute the tunneling time of the experiment. As a result, even though these two models have not much in common other than the energy-time uncertainty, results given here in Section 4.1 and in [52] show good agreement for He ionization in attosecond experiments. It is with further experimental data that possible relationship between our approach and that of [52] may be settled.

In Section 2 below given are derivation of the entropy characterizing the tunneling particle and definition of the tunneling time. Section 3 compares the entropic tunneling time with other times. Section 4 is devoted to numerical study including laser-induced He ionization and electron transfer 


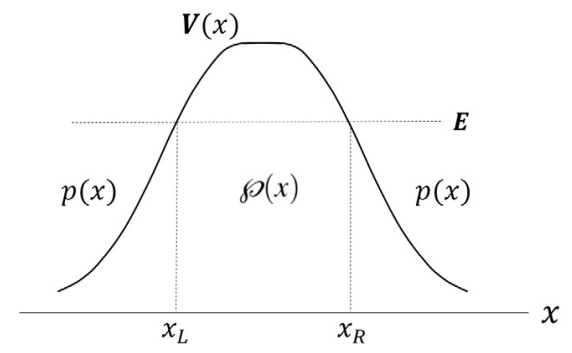

Fig. 1. Schematic illustration of the tunneling setup. Potential is smooth. Its central region is the tunneling region.

reactions in two separate subsections. Section 5 concludes the work and gives future prospects on applications to different tunneling-enabled phenomena and extensions to photon and phonon tunnelings.

\section{Entropic tunneling time}

In classical dynamics, time elapsed while a particle moves from one point to another cannot be determined without knowing its momentum at each point in between. This is because momentum is generator of the translation and, with strict energy conservation, it becomes $p(x)=\sqrt{2 m(E-V(x))}$ for a particle moving along $x$ axis with mass $m$, potential energy $V(x)$ and total energy $E$. The particle turns back to its region of incidence from the turning points $x_{L}$ and $x_{R}$ at which $E=V\left(x_{L}\right)=V\left(x_{R}\right)$. The setup is illustrated in Fig. 1. In this forbidden region (the central region in the figure) $E<V(x)$ and classical dynamics proceeds with imaginary momentum $p(x)=i \wp(x)$ with

$$
\wp(x)=\sqrt{2 m(V(x)-E)}
$$

and the lapse time it defines becomes also imaginary $t \rightarrow-i \tau_{c}$ (see [53] for time arrow) with

$$
\tau_{c}=\int_{x_{L}}^{x_{R}} \frac{m d x}{\wp(x)}
$$

defining what one may call the classical tunneling time. Its imaginary nature implies that traversing the classically forbidden region costs no real time. The scattering process seems instantaneous and acausal. There is an ongoing debate $[22,23,54]$ on the nature of the tunneling time. In the present work, assumption is that tunneling time is real and finite [55-57] and is consistent with earlier discussions in [58-60].

By definition, passage of the particle from $x_{L}$ to $x_{R}$ with conserved energy $(E<V(x))$ defines quantum tunneling. It is a pure quantum phenomenon. Tunneling time, however, is not a quantum concept [61]. The reason is that time is not an observable representable by some operator as otherwise it would stop flowing in its eigenstates. Physically correct description of tunneling time, a deterministic dimension, might therefore involve an amalgamate of the classical description above and the quantum behavior. (Despite these, recently Bauer proposed a self-adjoint time operator based on Dirac's formulation of relativistic quantum mechanics [62,63]. This proposed time is correlated with zitterbewegung type fluctuations, and has been claimed [64] to agree the experiment [51]. Loss of probability interpretation in relativistic realm and averaging-out of the zitterbewegung term over positive-energy states make this time definition curious.) To this end, one first notes that time flow is directly correlated with particle's momentum (as defined in Eq. (2)), and thus, tunneling time must be addressed in momentum eigenstates $\psi_{m}(x)$, not in energy eigenstates $\psi_{e}(x)$ (Schroedinger equation refers to energy not displacement). The second point is that, as a means of ensuring penetration of the particle into the classically forbidden region, classical momentum must function as the momentum eigenvalue associated with $\psi_{m}(x)$. More precisely, $\psi_{m}(x)$ must satisfy the eigenvalue 
equation $\hat{p} \psi_{m}(x)=\sqrt{2 m(E-V(x))} \psi_{m}(x)$ where $\hat{p}=-i \hbar \frac{d}{d x}$ is the momentum operator. In tunneling region, where $p(x)=i \wp(x)$, one gets

$$
\frac{d}{d x} \psi_{m}(x)=-\frac{\sqrt{2 m(E-V(x))}}{i \hbar} \psi_{m}(x) \equiv-\frac{\wp(x)}{\hbar} \psi_{m}(x)
$$

in agreement with energy conservation. This momentum eigenvalue shows that particle's momentum inside the tunneling region is strongly correlated with the potential function. At every point under the barrier, particle's momentum changes point to point and leads to the classical time in (2) integrated over the history of motion. This equation can always be integrated to find

$$
\psi_{m}(x)=\psi_{m}\left(x_{L}\right) \exp \left\{-\frac{1}{\hbar} \int_{x_{L}}^{x} \wp(\tilde{x}) d \tilde{x}\right\}
$$

which is an evanescent wave that decays exponentially as the particle penetrates farther and farther from $x_{L}$. This evanescent behavior is the key aspect of the tunneling phenomenon. It encodes all the essential ingredients needed to describe the tunneling dynamics. To ensure that particle is in the tunneling region, the probability to find the particle at $x$ satisfying $x_{L} \leq x \leq x_{R}$ (proportional to $\left.\psi_{m}^{\dagger}(x) \psi_{m}(x)\right)$ can be normalized with respect to the probability that it got into the tunneling region at $x_{L}$ (proportional to $\psi_{m}^{\dagger}\left(x_{L}\right) \psi_{m}\left(x_{L}\right)$ ). This way, probability to find the particle at $x=x_{R}$ becomes

$$
p_{m}=\frac{\psi_{m}^{\dagger}\left(x_{R}\right) \psi_{m}\left(x_{R}\right)}{\psi_{m}^{\dagger}\left(x_{L}\right) \psi_{m}\left(x_{L}\right)}=\exp \left\{-\frac{2}{\hbar} \int_{x_{L}}^{x_{R}} \wp(x) d x\right\} \equiv e^{-2 \Phi}
$$

where, for future use, one introduces the dimensionless quantity

$$
\Phi=\frac{1}{\hbar} \int_{x_{L}}^{x_{R}} \wp(x) d x
$$

which measures action of the particle in units of $\hbar$. It now becomes clear that $p_{m}$ vanishes for infinitely wide and infinitely high potential barriers $\left(p_{m} \rightarrow 0\right.$ as $\left.\Phi \rightarrow \infty\right)$ and equals unity if the barrier is absent $\left(p_{m} \rightarrow 1\right.$ as $\left.\Phi \rightarrow 0\right)$. Nevertheless, pertaining to a definite momentum state, it cannot tell whether tunneling has really been completed or not. The question of whether the particle has tunneled or reflected is determined by the tunneling transmission probability $p_{t}$ not $p_{m}$. It is obtained by solving the Schroedinger equation

$$
\frac{d^{2}}{d x^{2}} \psi_{e}(x)=-\frac{2 m}{\hbar^{2}}(E-V(x)) \psi_{e}(x) \equiv\left(\frac{\wp(x)}{\hbar}\right)^{2} \psi_{e}(x)
$$

wherein the energy eigenfunction $\psi_{e}(x)$, unlike the momentum eigenfunction $\psi_{m}(x)$, involves both right-evanescing and left-evanescing waves. They give rise to the well-known transmission and reflection probabilities [3,9,12].

In general, when interpreted as inverse temperature, the imaginary time is known to transform propagators in quantum mechanics into partition functions in statistical mechanics [65]. This ensures that tunneling time can be addressed in a statistical framework despite the peculiarity that what is referred to here is a single particle (not a collection of particles as in the usual statistical thermodynamics). Moreover, the particle is not in a mixed state (it is the evanescent wave decaying towards $x_{R}$ ). This means that entropy, required by temperature, must be defined in a different way. Fundamentally, entropy is given by logarithm of the number of microstates. The requisite microstates can be identified by excogitating to volume of the particle's phase space in units of $\hbar$. This quantity is precisely the main variable $\Phi$ and counting it means essentially the Bohr-Sommerfeld quantization rule. It refers to periodic dynamics which is what happens in tunneling region when potential is effectively inverted with imaginary time. Fig. 2 gives an illustration of how $\Phi$ can be related to the microstates pertaining to a tunneling particle. Identification of $\Phi$ with the number of microstates enables a statistical description of tunneling dynamics. For a correct formulation, one observes that entropy, specific to the tunneling region, must vanish when the barrier is absent $(\Phi \rightarrow 0)$. This means that the number of microstates can be taken as $2 \Phi+1$, where the factor of 2 is put for getting an integer since $\Phi$ is half-integer and the 1 is added for obtaining correct limiting value as $\Phi \rightarrow 0$. As a result, 


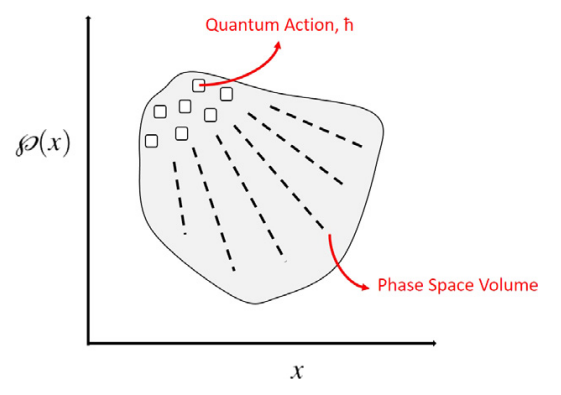

Fig. 2. Schematic representation of the phase space volume and total quantum actions.

the tunneling particle acquires entropy [66] $k_{B} p_{m} \log (1+2 \Phi)$ which equals, after relating $\Phi$ to $p_{m}$ via (5), the compact expression

$$
S\left(p_{m}\right)=k_{B} p_{m} \log \left(1-\log p_{m}\right)
$$

which satisfies $S\left(p_{m}=0\right)=0, S\left(p_{m}=1\right)=0$ and $S\left(p_{m}\right) \geq 0$. Needless to say, this loglog structure is an artifact of the exponential relationship between $\Phi$ and $p_{m}$ in Eq. (5). To see this, one notes that use of the uniform probability $p_{u}=1 /(1+2 \Phi)$ would result in the familiar Boltzmann entropy $-k_{B} p_{u} \log p_{u}$ in place of the loglog entropy in (8). In consequence, the entropy formula (8) is specific to the tunneling region and gives a statistical description of its imaginary-time and evanescent dynamics.

In this statistical formulation, the energy $E$ of the particle functions as the total internal energy. The thermal energy, on the other hand, equals the entropy rate of change of the internal energy. This is so because tunneling involves a single particle with quantum probabilistic qualities, and the energy rate of change of the tunneling entropy in (8) gives

$$
\frac{1}{k_{B} T} \equiv \frac{1}{k_{B}} \frac{\partial S\left(p_{m}\right)}{\partial E}=-\frac{2 \tau_{c}}{\hbar} e^{-2 \Phi}\left[\frac{1}{1+2 \Phi}+\log \frac{1}{1+2 \Phi}\right]
$$

where $\tau_{c}$ is the classical tunneling time in (2) and is $\tau_{c}=-\hbar \partial \Phi / \partial E$. It is not surprising that the temperature $T$ is proportional to the reciprocal of $\tau_{c}$ [65]. This temperature, defined for a single particle owing to its quantum indeterminacy, involves both the Boltzmann constant $k_{B}$ and Planck constant $\hbar$. In a true thermodynamical system there can exist no $\hbar$ sensitivity in the classical limit. The specialty of tunneling is that it is a pure quantum phenomenon having no classical limit. Thus, the statistical description of tunneling we are presenting necessarily involves both $k_{B}$ and $\hbar$.

In quantum tunneling, particle's energy $E$ stays constant throughout the barrier, and particle ionizes to continuum with the same energy $E$ at the end of tunneling. (In experimental environments, time measurement after ionization can alter energy $E$. Such effects do not influence tunneling time definitions as they all refer to the barrier region. This is valid also for the ETT.) In contrast to $E$, however, the thermal energy $k_{B} T$ varies with barrier shape as in (9) and, physically, it sets a finite time interval $\Delta t$ in the philosophy of the energy-time uncertainty product. This time interval, as insured by construction of the probability $p_{m}$ in (5), must be nothing but the time elapsed while the particle gets from $x_{L}$ to $x_{R}$. One here notes that these turning points vary with $E$ and, in general, lower the $E$ larger the $x_{R}-x_{L}$ and longer the tunneling duration. Its dependence on $p_{m}$ suggests that $\Delta t$ can be related to tunneling duration. In this sense, let us assume that this is some sort of a tunneling time, and study the consequences of this assumption. Thus, one defines tunneling time as

$$
\Delta t=\frac{\hbar}{2 \Delta E_{\text {ther }}}
$$

which is no different than the energy-time uncertainty relation. In here, $\Delta E_{\text {ther }}$ is the thermal energy needed for completing the tunneling, and it can be written as

$$
\Delta E_{\text {ther }}=p_{t}\left(2 \pi k_{B} T\right)
$$


in view of the finite-temperature quantum fluctuations. Here, $p_{t}$ is the tunneling transmission probability (computed from solution $\psi_{e}$ of the Schroedinger equation). The thermal energy $2 \pi k_{B} T$ might be interpreted as the splitting between Matsubara levels [67,68] in finite-temperature quantum theory. At last, the tunneling time in (10), after replacing $k_{B} T$ values from Eq. (9), takes the general form

$$
\Delta t=\frac{\hbar}{2 p_{t}\left(2 \pi k_{B} T\right)}=-\frac{\tau_{c}}{2 \pi p_{t}} e^{-2 \Phi}\left(\frac{1}{1+2 \Phi}+\log \frac{1}{1+2 \Phi}\right)
$$

valid for any particle and any smooth potential. The tunneling time formula (12), will be hereon called entropic tunneling time (ETT) to distinguish it from other tunneling time definitions like phase time Eq. (17) and dwell time Eq. (18), which exist in the literature.

Before closing, it proves complementary to discuss the nature of the ETT. In the classification of Busch $[69,70]$, it is an intrinsic dynamical time. The reason is that it is controlled by particle's underbarrier momentum $\wp(x)$, which is integrated over the barrier region to form $\Phi$. However, one must pay attention that the energy-time uncertainty relation in (10) is defined between $\Delta t$ and the thermal energy $\Delta E_{\text {ther }}$ in (11) (not the fluctuations in the true energy $E$ of the particle, which is held constant during the entire tunneling dynamics). In this sense, though it is an intrinsic dynamical time, the ETT can be contrasted with external time measurements (as in Section 4 below) as it does not necessitate any fluctuations in $E$ under the barrier [69,70].

\section{Comparing ETT with other times}

There are already manifold time definitions in the literature [28-31]. They vary in their origins, formulations and predictions. Two of them, the Larmor and Buttiker-Landauer times, are special in that they are defined via not the potential $V(x)$ alone but with its purposive modifications. The Larmor time is based on the Larmor precession of spin when the classically forbidden region is covered by an external magnetic field [36-38]. The Buttiker-Landauer time is defined via an oscillating barrier [32-34]. These two times have been argued [35,71] to be not the means but deviations of the tunneling time distributions. They have obviously nothing in common with the ETT, which is based on the potential barrier $V(x)$ alone. On the other hand, two well-known time definitions, the phase time [28-31,40-42] and the dwell time [28-31,36], which, just like the ETT, are based on the potential energy $V(x)$. They do not involve any external agents like the magnetic field used in Larmor time. These three time definitions: ETT, phase, and dwell, having a common setup, can thus be directly contrasted to determine their physical relevance. To this end, as a simple setup, one can consider a rectangular potential barrier. (One must, however, keep in mind that a rectangular barrier does not quite fit to the WKB criteria. Nevertheless, it provides a viable framework to compare the time definitions. In case of worry, it can be approximated through a steep tanh potential.) Then, for a barrier of height $V_{0}$ and width $L$, one derives the tunneling transmission probability

$$
p_{t}^{\square}=\frac{1}{1+\frac{V_{0}^{2} \sinh ^{2} \Phi}{4 E\left(V_{0}-E\right)}}
$$

where $\square$ symbolizes the rectangular potential. With this transmission probability, the ETT takes the form

$$
\begin{aligned}
(\Delta t)_{E T T}^{\square}=-\frac{\tau_{c}^{\square}}{2 \pi\left(V_{0}-E\right)}( & \left.\left(V_{0}-E\right)+\frac{\left(V_{0} \sinh \Phi^{\square}\right)^{2}}{4 E}\right) e^{-2 \Phi^{\square}} \\
& \times\left(\frac{1}{1+2 \Phi^{\square}}+\log \frac{1}{1+2 \Phi^{\square}}\right)
\end{aligned}
$$

in which

$$
\Phi^{\square}=\frac{\sqrt{2 m\left(V_{0}-E\right)}}{\hbar} L
$$


is the abbreviated action in the classically forbidden region as follows from (6), and

$$
\tau_{c}^{\square}=\frac{m L^{2}}{\hbar \Phi^{\square}}
$$

is the traversing time $-i \tau^{\square}$ of the particle obeying classical motion laws as follows from its definition in (2), and $L=x_{R}-x_{L}$ is the barrier width.

The phase time (designated by $\varphi$ ), deriving from the stationarity of the phase of the transmission amplitude [28-31,40-42], takes the form

$$
\begin{aligned}
(\Delta t)_{\varphi}^{\square}= & \frac{\left(\tau_{c}^{\square}\right)\left(p_{t}^{\square}\right)}{2\left(\Phi^{\square}\right)^{2} \Phi_{E}^{3}}\left[\Phi^{\square} \Phi_{E}^{2}\left(\left(\Phi^{\square}\right)^{2}-\Phi_{E}^{2}\right)\right. \\
& \left.+\left(\left(\Phi^{\square}\right)^{2}+\Phi_{E}^{2}\right)^{2} \sinh \Phi^{\square} \cosh \Phi^{\square}\right]
\end{aligned}
$$

where $\Phi_{E}=\sqrt{2 m E L^{2}} / \hbar$. In this phase time, the hyperbolic functions arise from the phase of the transmission amplitude.

The dwell time, expressing how long the particle stays in the barrier region [28-31,36], is given by

$$
\begin{aligned}
(\Delta t)_{D}^{\square} & =\frac{\left(\tau_{c}^{\square}\right)\left(p_{t}^{\square}\right)}{2\left(\Phi^{\square}\right)^{2} \Phi_{E}}\left[\Phi^{\square}\left(\left(\Phi^{\square}\right)^{2}-\Phi_{E}^{2}\right)\right. \\
& \left.+\left(\left(\Phi^{\square}\right)^{2}+\Phi_{E}^{2}\right) \sinh \Phi^{\square} \cosh \Phi^{\square}\right]
\end{aligned}
$$

where, compared to the phase time, lower powers of $\Phi^{\square}$ and $\Phi_{E}$ are involved.

The three tunneling times the ETT (denoted by $(\Delta t)_{E T T}^{\square}$ ), the phase time (denoted by $(\Delta t)_{\varphi}^{\square}$ ) and the dwell time (denoted by $(\Delta t)_{D}^{\square}$ ) are seen to be distinct functions. They lead thus to different predictions for time spent during tunneling. Nevertheless, to reveal their physical relevance it is convenient to compare them for wide potentials $(L \rightarrow \infty)$ :

$$
\begin{aligned}
(\Delta t)_{E T T}^{\square} & \rightarrow \infty \\
(\Delta t)_{\varphi}^{\square} & \rightarrow \frac{\hbar}{E} \sqrt{\frac{E}{V_{0}-E}} \\
(\Delta t)_{D}^{\square} & \rightarrow \frac{\hbar}{V_{0}} \sqrt{\frac{E}{V_{0}-E}}
\end{aligned}
$$

where the ETT is seen to diverge as expected of a potential barrier of infinite width. The phase and dwell times, however, give the unphysical result that it takes a finite time to traverse an infinitely wide potential barrier. These two times suffer from superluminality. More strikingly, those finite times vanish as $V_{0} \rightarrow \infty$, meaning that the particle tunnels through an infinitely wide and infinitely high potential barrier instantaneously. This effect, the Hartman effect [72], renders the phase and dwell times unphysical. Needless to say, the ETT is subluminal and suffers from no unphysical aspects like the Hartman effect. Moreover, relationship of tunneling time to particle's dynamical transport has been verified for electrons in [73] and discussed by Kullie in [74].

Furthermore, apart from tunneling time definitions above, the complex tunneling times are hard to make sense [75]. The path integral averages of the classical time [43-45] and of the Larmor time [76,77] give rise to complex times. They also arise via scattering-theoretic formulation [39]. Their real and imaginary parts are related to other tunneling times in specific ways [28-31,35]. Unlike them, the ETT is purely real and bears no relation to complex times.

\section{Confronting ETT with experiment}

In this section, we shall perform numerical analysis to test the ETT against certain experimental results. 


\subsection{Laser-driven He ionization}

Electric fields of high-intensity lasers reshape Coulomb potential in atoms to form a potential barrier through which electrons can tunnel to continuum [26,27]. At the peak value $\mathcal{E}$ of the electric field, one of the electrons in He possesses the effective potential energy

$$
V(x)=-\frac{Z_{\text {eff }}}{x}-\mathcal{E} x
$$

which is parametrized in terms of an effective nuclear charge $Z_{\text {eff }}$. The transmission probability takes the form [78,79]

$$
p_{t}^{H e}=\frac{1}{\cosh ^{2} \Phi}
$$

after solving (7) and matching the solutions at each of the turning points $x_{L, R}$. With this transmission probability, ETT (12) becomes

$$
(\Delta t)^{H e}=-\frac{\tau_{c}}{2 \pi} \cosh ^{2} \Phi e^{-2 \Phi}\left(\frac{1}{1+2 \Phi}+\log \frac{1}{1+2 \Phi}\right) .
$$

On the other hand, advancements in ultrafast science, where strong laser fields are used to ionize atoms by quantum tunneling, are capable of observing tunneling transition and measuring the tunneling time [15-21]. In spite of various factors affecting the experiments [22-25], improving on previous single-particle tunneling time measurements [20] by using attoclock in strong laser fields [21], in 2013 the research team of Ursula Keller at ETH Zurich have performed a refined measurement of the tunneling time of electrons in He atom [51]. Moreover, their measurements have been shown to be stable [80] (see also the simulation study [81]) against non-adiabatic effects [22,23]. As a result, using the effective potential (22) enables us to use this experiment as a testbed for the ETT. The limiting factor here is validity of the WKB solution. The WKB approximation holds good for smooth potentials. The same is not true for steep potentials with sharp edges. For such potentials, beyondthe-WKB effects can be significant. For the He ionization problem at hand, the WKB approximation hardly works at large laser field strength $\mathcal{E}$ for which potential is steep (see the potentials in [82]). In such regions, agreement with experiment can require beyond-the-WKB effects to be incorporated. Besides, one keeps in mind that the laser field, depending on its strength, can cause either multiphoton or tunneling ionization. And a sensible comparison of the ETT with experiments is possible only in tunneling regime corresponding to the Keldysh parameter [26,27] range $\gamma \lesssim 1$.

In the experimental setup, laser intensity $3.478 \times 10^{16} \mathrm{~W} / \mathrm{cm}^{2} \mathcal{E}^{2}$ is varied from $0.730 \times 10^{14}$ to $7.50 \times 10^{14} \mathrm{~W} / \mathrm{cm}^{2}$ by varying the peak electric field $\mathcal{E}$ from 0.04 to 0.11 in atomic units. The electron energy $E=-0.904$ a.u. is the first ionization potential of the He atom. Momentum distribution of the liberated electrons are obtained by cold-target recoil-ion momentum spectrometer (COLTRIMS) and by velocity map imaging spectrometer (VMIS) (see the experiment section of [51] for details). The VMIS is used particularly at low laser intensities. Quantum tunneling is ensured to be the dominant ionization mechanism by keeping the Keldysh parameter small $(\gamma \lesssim 1)$. The experimental results are given in Fig. 3 of [51]. Depicted in Fig. 3(a) of [51] are different tunneling times [32,36,39,40,43-45] contrasted with experiment's own results. Similarly, given in Fig. 3(b) and (d) of [51], are tunneling times as functions of the peak electric field $\mathcal{E}$ and barrier width (approximated as $E / \mathcal{E}$ a.u. in the experiment). The experiment (as well as [80]) also indicates that among all widely-used tunneling time definitions only the FPI time comes closest to its measurements (see Fig. 3(b) and (d) of [51]). Additionally, [83] also indicates that the phase and dwell times both overestimate the experimental result [51].

For confronting the ETT (24) with experiment, it suffices to replace the potential energy (22) in $\Phi$, and $\tau_{c}$, and evaluate them with the turning points $x_{L}$ and $x_{R}>x_{L}$ satisfying

$$
x_{L(R)}=\frac{E-(+) \sqrt{E^{2}-4 Z_{e f f}\left(x_{L(R) \mathcal{E}}\right.}}{2 \mathcal{E}}
$$

which are the roots of the vanishing kinetic energy condition $V(x)-E=0$. It is clear that better the knowledge of $Z_{\text {eff }}$ better the prediction of tunneling time (through the turning points and hence $\Phi$ ). Following the literature, three different $Z_{\text {eff }}$ : 
1. SAE potential $[84]$

$$
Z_{\text {eff }}=Z+a_{1} e^{-a_{2} x}+a_{3} x e^{-a_{4} x}+a_{5} e^{-a_{6} x}
$$

in which $Z=1, a_{1}=1.231, a_{2}=0.662, a_{3}=-1.325, a_{4}=1.236, a_{5}=-0.231$, and $a_{6}=0.480$ in atomic units.

2. Kullie constant $[52,85] Z_{\text {eff }}=1.375$, and

3. Clementi et al. constant [86] $Z_{\text {eff }}=1.6875$.

were used here. The turning points in (25) are directly evaluated for constant $Z_{\text {eff }}$. For the SAE potential, however, it is necessary to find a self-consistent solution for $x_{L, R}$. This is done by starting with a random $x$ value and iterating it $N$ times until $V\left(x_{N}\right)-E<10^{-4}$. We list down $x_{L}$ and $x_{R}$ values and the corresponding classical time $\tau_{c}$ and the ETT in Table 1 by considering two peak values for the laser field. The ETT for different $Z_{\text {eff }}$ are plotted in Fig. 3 as functions of the peak electric field $\mathcal{E}$ and experiment's barrier width $E / \mathcal{E}$ (which is not the true barrier width $x_{R}-x_{L}$ ). It is superimposed on Fig. 3(b) and (d) of [51]. The figure manifestly shows that ETT exhibits good agreement with the experimental data for all three potential models. They, nevertheless, start differing at large laser field $\mathcal{E}$ values. This can be understood as follows:

1. At small $\mathcal{E}$, the potential $V(x)$, away from the nucleus, is smooth in all three cases (SAE, Kullie and Clementi). It supports the WKB solution. This is confirmed by the fact that ETT(SAE), ETT(Kullie) and ETT(Clementi) fairly agree at low laser field values $\mathcal{E}$ (see Fig. 3 below $\mathcal{E} \simeq 0.08$ ).

2. At large $\mathcal{E}$, the three times start differing significantly. The reason for this is that potential $V(x)$ is no longer smooth, and depending on the potential parameters the ETT values diverge. The potential takes a right-triangular shape with smaller and smaller depression angle for larger and larger $\mathcal{E}$. The WKB solution hardly works for this potential (see the detailed analysis in [82]). To have a quantitative understanding of the ETT curves at large $\mathcal{E}$, it suffices to consider a righttriangle potential $V(x)=V_{0}-\mathcal{E} x$ extending from $x=0$ to $L$ (having the same width as the rectangular potential in Section 3$)$ for which one gets

$$
\Phi^{\triangle}=\frac{2}{3} \frac{\left(V_{0}-E\right)}{\mathcal{E} L} \Phi^{\square}
$$

and

$$
\tau_{c}^{\triangle}=\frac{2\left(V_{0}-E\right)}{\mathcal{E} L} \tau_{c}^{\square}
$$

so that $p_{m}^{\Delta}>p_{m}^{\square}$ and $p_{t}^{\Delta}>p_{t}^{\square}$. Using these in the general ETT formula in (12) one can compute tunneling time for the triangular potential. It is clear that $\Phi^{\Delta}<\Phi^{\square}$ and $\tau_{c}^{\Delta}<\tau_{c}^{\square}$ under sufficiently strong laser fields i.e. large $\mathcal{E}$. This means that the ETT curves in Fig. 3 will be pushed down depending on the laser field strength. In fact, the classical time (28) is already sufficient to understand this. The simple triangular potential, though different than the actual potential in (22), is powerful enough to reveal the essential features of the ETT curves in Fig. 3.

3. All might seem fine, but one must still keep in mind that the triangular potential (like the He ionization at large $\mathcal{E}$ ) is sensitive to beyond-the-WKB effects. The WKB-based ETT predictions may not therefore be accurate. Indeed, the ETT(Clementi), for instance, takes smaller values than expected (though $Z_{\text {eff }}=1.6875$ is reasonable in the large $\mathcal{E}$ domain). This can be understood as an artifact of the beyond-the-WKB effects. This problem, as discussed also in detail in [82], is a characteristic feature of large $\mathcal{E}$ region.

These properties help interpreting the ETT curves in Fig. 3 as a transition from smooth to steep potential regions.

Of all the time definitions available, as shown in Fig. 3, only the FPI time [43-45,50] comes closest to the experimental data. Indeed, it exhibits good agreement with the experimental data in both panels. Yet, in the top panel, its predictions diverge from the data as the peak electric field increases. In the bottom panel, it matches with the COLTRIMS data at low barrier widths while it diverges at 

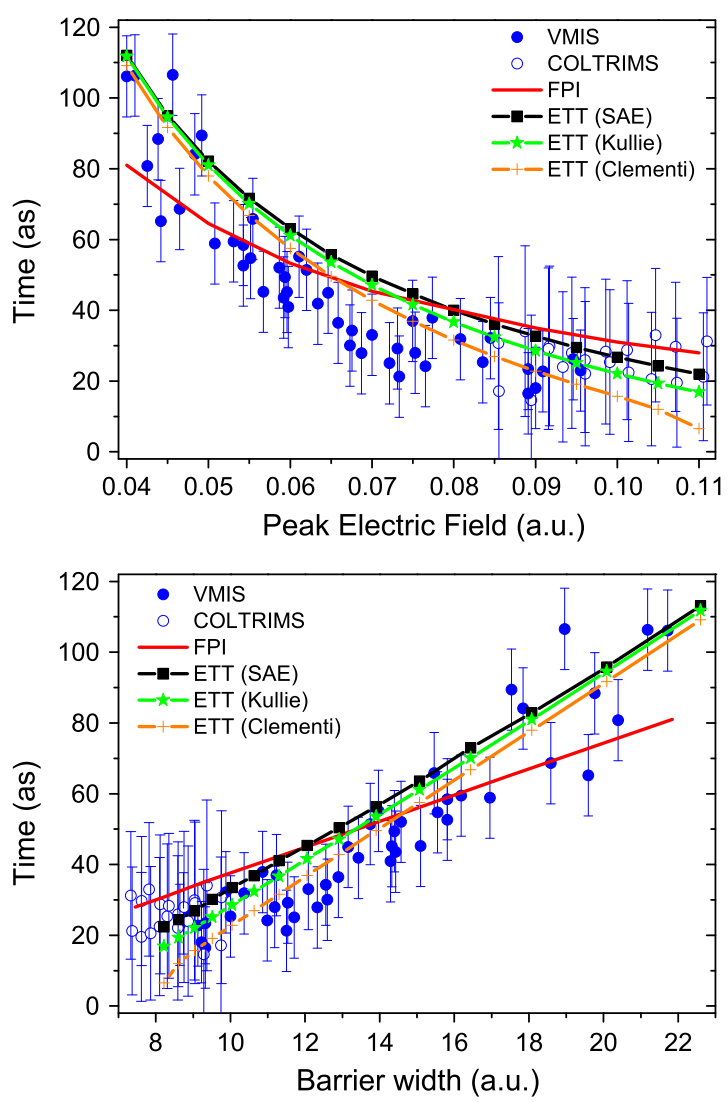

Fig. 3. Tunneling times as functions of peak electric field $\mathcal{E}$ (top panel) and experimental barrier width $(E / \mathcal{E}$ ) (bottom panel). The entropic tunneling time (ETT) is depicted by filled square, star and plus representing SAE, Kullie and Clementi et al. models, respectively. It is seen to agree with the experimental data throughout. Both panels explicitly show how the entropic time adheres to the experimental data [51]) while the FPI time diverges away from data at the asymptotics.

Table 1

Contrasting the three $Z_{\text {eff }}$ models, after setting $\mathcal{E}=0.04$ a.u. ( $\mathcal{E}=0.11$ a.u.), in terms of their predictions for the turning points $x_{L, R}$, classical time $\tau_{c}$ and the ETT.

\begin{tabular}{lllll}
\hline & $x_{L}$ (a.u.) & $x_{R}$ (a.u.) & $\tau_{c}$ (as) & ETT (as) \\
\hline SAE & $1.24(1.39)$ & $21.43(6.90)$ & $833.82(312.24)$ & $113.08(22.20)$ \\
Kullie & $1.64(2.02)$ & $20.96(6.20)$ & $850.73(322.72)$ & $111.75(16.85)$ \\
Clementi & $2.05(2.87)$ & $20.55(5.35)$ & $856.49(326.50)$ & $109.14(6.54)$ \\
\hline
\end{tabular}

larger barrier widths. In contrast to these divergent behaviors in the FPI time, ETT stays congruent to experimental data for a fairly wide range of potential parameters.

As a result, it would not be unrealistic to conclude that the ETT shows good agreement with experiment, and furthermore, outperforms the widely-used tunneling time models among those shown in Fig. 3(a) of [51] and the FPI time.

\subsection{Electron transfer reactions}

Rectangular potential barriers, apart from their direct solubility, prove useful in modeling tunneling systems whose potential barriers are nearly constant. Even though WKB approach does not 

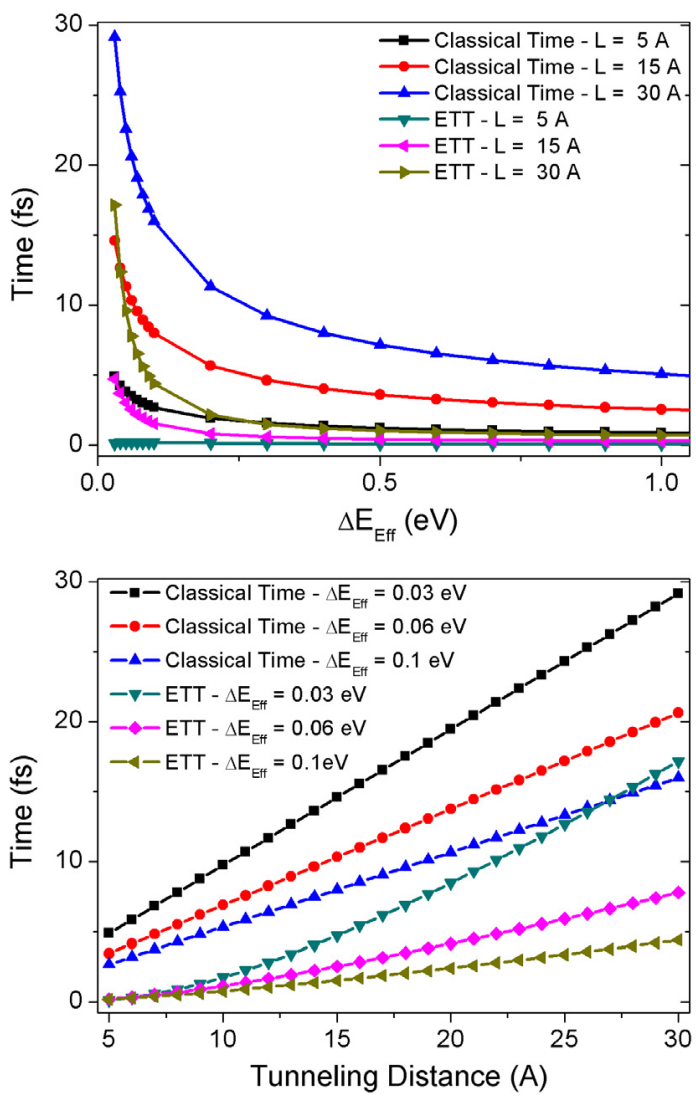

Fig. 4. Electron transition time as functions of tunneling distance given for different $\Delta E_{E f f}$ values (top panel) and $\Delta E_{E f f}$ given for different tunneling distance values (bottom panel) calculated in terms of both classical time and ETT. Energy of an electron $E$ is taken as $1 \mathrm{eV}$.

work properly for this potential barrier shape, as already mentioned, one can still expected to obtain corroborative results. As an example, efficient tunneling barrier $\left(\Delta E_{E f f}=V_{0}-E\right)$ proves to be a useful approach in the case of long-range electron transfer reactions [87]. The transmission probability and ETT of this simple case is given in Eqs. (13) and (14) respectively. Notice that, the exact transmission probability (13) reduces to the WKB result in (23) only when $E=V_{0} / 2$.

In the case of electron transfer reactions, typical range for an electron to transit from donor to acceptor is in between 5 and $30(\AA)$ (the range of electron transfer in proteins from 15 to 30 (Å) [88]). Nuclear vibrations have typically $<3000 \mathrm{~cm}^{-1}$ frequency range. Corresponding half-period of vibrations are thus above the 5 femtosecond (fs) scale. Using ETT and classical time in (15), electron transition times can be calculated. It is found that, classical time is comparable to the nuclear vibrations in Fig. 4. On the other hand, ETT gives lower values compared to classical time, in both cases. From this, one recalls that $\tau_{c}$ is the absolute value of the imaginary time spent during the classical motion. The difference between the two tunneling times indicates how strong the quantum effects are. Furthermore, it is seen that these values of tunneling time are even lower than half period of the corresponding nuclear vibrations, whereby proving the predomination of the adiabatic region [13]. However, it is for the $\Delta E_{E f f}<0.1 \mathrm{eV}$ and the tunneling distance $>15(\AA)$ that the tunneling time becomes comparable to the time scale of nuclear vibrations. Time scale compatibility between nuclear vibrations and tunneling time allows overlap of molecular configuration change with the electron transition process while electron is tunneling. Due to energy conservation, configuration 
change during the electron tunneling leads to an energy exchange between electron and nuclei, which entangles them. Therefore, in this restricted region with time scale comparability, it becomes possible for an electron to make transition from $\mathrm{BO}$ to non-BO regime where acceptor-donor wavefunctions collapse. Similar analysis was performed already in [14], where their tunneling time definition for simple rectangular potential barrier equals to the classical time definition in (15). It follows the same trend with classical time given in Fig. 4. It obviously lacks quantum contributions and proves thus insufficient for having a clear picture of $\mathrm{BO}$ to non-BO transition regime. As a result, the conditions determined by ETT set the range of energy difference and tunneling distance more accurately for long range electron transfer reactions to take place.

\section{Conclusion and future prospects}

ETT, with its statistical conception, subluminal nature and experimental confirmation, works through as a realistic model of the tunneling time. It provides a quantum theoretic framework by which one can analyze all kinds of tunneling-enabled phenomena ranging from STM to DNA mutation and flash memory to interstellar chemistry. This rather widespread role facilitates phenomenological tests and possible improvements of the entropic formalism through variety of sources.

The tunnel effect, a manifestation of the evanescent wave behavior, can occur in all wave phenomena. A generic wave equation

$$
\frac{d^{2}}{d x^{2}} W(x)=-k^{2}(x) W(x)
$$

portrays a propagating wave for $k(x) \in \Re$ and evanescing wave for $k(x)=i \kappa(x) \in \mathfrak{I}$. Pragmatically, ETT formalism can be extended to this wave behavior with the identification

$$
\frac{\wp(x)}{\hbar} \rightarrow \kappa(x)
$$

as revealed by contrasting (29) with (7). To make sense of this formal equivalence, it is necessary to determine first the origin of the imaginary, inhomogeneous wavenumber $\kappa(x)$ in view of (1). Indeed, monochromatic wave must have a frequency below the natural cut-off frequency of the medium for evanescent behavior to occur. Next, it is necessary to construct the quanta corresponding to the wave so that evanescing characterizes the tunneling phenomenon. Finally, it is necessary to establish an analogy with the Schroedinger equation by taking into account the symmetries of the wave equation.

There are numerous wave phenomena. The probability waves of quantum theory, $W(x) \equiv \psi_{e}(x)$, govern electron tunneling in semiconductors, Hydrogen tunneling in biochemical systems and Helium tunneling in nuclear systems. The electric waves, $W(x) \equiv \mathcal{E}(x)$, describe photon tunneling in materials with imaginary refractive index (band gaps, dielectric gaps, air gaps) [89-91]. The photonic STM [9294], scanning of surfaces with a fiber optic tip, is a direct application of photon tunneling. The sound waves, on the other hand, encode phonon tunneling through acoustic band gaps [95-98]. Tunneling of the thermal vibrations of an STM tip to the sample is a direct realization of the phonon tunneling [99]. The optical and acoustic tunneling studies have been thoroughly reviewed in [100] experiment by experiment. The photon and phonon tunneling processes, interpreted so far only with phase and dwell times [100], need be analyzed and reinterpreted within ETT formalism, as is being planned to be done in upcoming work.

The ETT is new. It is theoretically consistent and experimentally pertinent for smooth potentials. In fact, one direction to improve on the present work would be to include of subleading WKB corrections (involving derivatives of the potential). Besides, the ETT can be tested against experimental data on appropriate physical, chemical and biological processes.

\section{Acknowledgments}

The authors thank Onur Rauf Yılmaz for reading the manuscript. They are grateful to Dr. Ossama Kullie for useful e-mail exchange and enlightening comments. They thank A. Landsman (through C. Hofmann) for the experimental data used in Fig. 3. The authors thank contentious referee for constructive suggestions. 


\section{References}

[1] G. Gamow, Nature 122 (1928) 805-806.

[2] E. Condon, P. Morse, Rev. Modern Phys. 3 (1) (1931) 43.

[3] L. MacColl, Phys. Rev. 40 (4) (1932) 621.

[4] L. Esaki, Phys. Rev. 109 (2) (1958) 603.

[5] L. Esaki, IEEE Trans. Electron Devices 23 (7) (1976) 644-647.

[6] G. Binnig, H. Rohrer, C. Gerber, E. Weibel, Phys. Rev. Lett. 49 (1) (1982) 57.

[7] O. Miyashita, M.Y. Okamura, J.N. Onuchic, Proc. Natl. Acad. Sci. USA 102 (10) (2005) 3558-3563.

[8] N. Nelson, W. Junge, Annu. Rev. Biochem. 84 (2015) 659-683.

[9] M. Razavy, Quantum Theory of Tunneling, Vol. 1222445599, World Scientific, 2003.

[10] R.J. McMahon, Science 299 (5608) (2003) 833-834.

[11] N. Lambert, Y.-N. Chen, Y.-C. Cheng, C.-M. Li, G.-Y. Chen, F. Nori, Nat. Phys. 9 (1) (2013) 10-18.

[12] D. Roy, Quantum Mechanical Tunnelling and its Applications, World Scientific, 1986.

[13] A. Nitzan, J. Jortner, J. Wilkie, A.L. Burin, M.A. Ratner, J. Phys. Chem. B 104 (24) (2000) 5661-5665.

[14] A. Stuchebrukhov, J. Phys. Chem. B 120 (8) (2015) 1408-1417.

[15] A.M. Steinberg, P.G. Kwiat, R.Y. Chiao, Phys. Rev. Lett. 71 (5) (1993) 708.

[16] M. Uiberacker, T. Uphues, M. Schultze, A.J. Verhoef, V. Yakovlev, M.F. Kling, J. Rauschenberger, N.M. Kabachnik, H. Schröder, M. Lezius, et al., Nature 446 (7136) (2007) 627-632.

[17] O. Smirnova, Y. Mairesse, S. Patchkovskii, N. Dudovich, D. Villeneuve, P. Corkum, M.Y. Ivanov, Nature 460 (7258) (2009) 972-977.

[18] O. Smirnova, S. Patchkovskii, Y. Mairesse, N. Dudovich, M.Y. Ivanov, Proc. Natl. Acad. Sci. 106 (39) (2009) $16556-16561$.

[19] S. Haessler, J. Caillat, W. Boutu, C. Giovanetti-Teixeira, T. Ruchon, T. Auguste, Z. Diveki, P. Breger, A. Maquet, B. Carré, et al., Nat. Phys. 6 (3) (2010) 200-206.

[20] P. Eckle, A. Pfeiffer, C. Cirelli, A. Staudte, R. Dörner, H. Muller, M. Büttiker, U. Keller, Science 322 (5907) (2008) 1525-1529.

[21] A.N. Pfeiffer, C. Cirelli, M. Smolarski, U. Keller, Chem. Phys. 414 (2013) 84-91.

[22] M. Lein, Nature 485 (7398) (2012) 313-314.

[23] L. Torlina, F. Morales, J. Kaushal, I. Ivanov, A. Kheifets, A. Zielinski, A. Scrinzi, H.G. Muller, S. Sukiasyan, M. Ivanov, et al., Nat. Phys. 11 (6) (2015) 503-508.

[24] D. Shafir, H. Soifer, B.D. Bruner, M. Dagan, Y. Mairesse, S. Patchkovskii, M.Y. Ivanov, O. Smirnova, N. Dudovich, Nature 485 (7398) (2012) 343-346.

[25] M. Sabbar, L. Gallmann, S. Heuser, M. Lucchini, U. Keller, R. Boge, C. Cirelli, Attochirp-corrected photo ionization time delays using coincidence attosecond streaking, 2014.

[26] L. Keldysh, Zh. Eksp. Teor. Fiz. 47 (1964).

[27] V.S. Popov, Phys.-Usp. 47 (9) (2004) 855-885.

[28] E. Hauge, J. Støvneng, Rev. Modern Phys. 61 (4) (1989) 917.

[29] V.S. Olkhovsky, E. Recami, Phys. Rep. 214 (6) (1992) 339-356.

[30] R. Landauer, T. Martin, Rev. Modern Phys. 66 (1)(1994) 217.

[31] G. Privitera, G. Salesi, V.S. Olkhovsky, E. Recami, Tunnelling times: an elementary introduction, ArXiv Preprint Quant-Ph/ 0412146, 2004.

[32] M. Büttiker, R. Landauer, Phys. Rev. Lett. 49 (23) (1982) 1739.

[33] M. Büttiker, R. Landauer, IBM J. Res. Dev. 30 (1986) 451.

[34] S. Boonchui, V. Sa-Yakanit, Phys. Rev. A 77 (4) (2008) 044101.

[35] N. Yamada, Phys. Rev. Lett. 93 (17) (2004) 170401.

[36] M. Büttiker, Phys. Rev. B 27 (10) (1983) 6178.

[37] V. Rybachenko, Sov. J. Nucl. Phys. 5 (1967) 484.

[38] J. Falck, E. Hauge, Phys. Rev. B 38 (5) (1988) 3287.

[39] E. Pollak, W.H. Miller, Phys. Rev. Lett. 53 (2)(1984) 115.

[40] E.P. Wigner, Phys. Rev. 98 (1) (1955) 145.

[41] S. Bandopadhyay, A. Jayannavar, Internat. J. Modern Phys. B 21 (10) (2007) 1681-1704.

[42] A.E. Bernardini, Ann. Physics 324 (6) (2009) 1303-1339.

[43] D. Sokolovski, L. Baskin, Phys. Rev. A 36 (10) (1987) 4604.

[44] H. Fertig, Phys. Rev. Lett. 65 (19) (1990) 2321.

[45] T. Martin, Internat. J. Modern Phys. B 10 (27) (1996) 3747-3763.

[46] H. Fertig, Phys. Rev. B 47 (3) (1993) 1346.

[47] D. Sokolovski, J. Connor, Phys. Rev. A 44 (3) (1991) 1500.

[48] D. Sokolovski, J. Connor, Phys. Rev. A 47 (6) (1993) 4677.

[49] D. Mugnai, A. Ranfagni, Il Nuovo Cimento D 14 (5) (1992) 541-551.

[50] D. Sokolovski, S. Brouard, J. Connor, Phys. Rev. A 50 (2) (1994) 1240.

[51] A.S. Landsman, M. Weger, J. Maurer, R. Boge, A. Ludwig, S. Heuser, C. Cirelli, L. Gallmann, U. Keller, Optica 1 (5) (2014) 343-349.

[52] O. Kullie, Phys. Rev. A 92 (5) (2015) 052118.

[53] H.D. Zeh, The Physical Basis of The Direction of Time, Springer Science \& Business Media, 2007.

[54] H. Ni, U. Saalmann, J.M. Rost, Phys. Rev. Lett. 117 (2016) 023002.

[55] R. Landauer, Nature 341 (1989) 567-568. 
[56] A.M. Steinberg, Phys. World 16 (12) (2003) 19.

[57] P.C.W. Davies, Am. J. Phys. 73 (1) (2005) 23-27.

[58] F.E. Low, P.F. Mende, Ann. Physics 210 (2) (1991) 380-387.

[59] D.A. Demir, Real-time tunneling, ArXiv Preprint Quant-Ph/9809036, 1998.

[60] F. Grossmann, Phys. Rev. Lett. 85 (5) (2000) 903.

[61] W. Pauli, in: H. Geiger, K. Scheel (Eds.), Handbuch der Physik, Vol. 23, first ed., Springer, Berlin, 1926.

[62] M. Bauer, Internat. J. Modern Phys. A 29 (06) (2014) 1450036.

[63] M. Bauer, The problem of time in quantum mechanics, ArXiv Preprint arXiv:1606.02618, 2016.

[64] M. Bauer, Tunneling in attosecond optical ionization and a dynamical time operator, ArXiv Preprint arXiv:1608.03492, 2016.

[65] R. Feynman, A. Hibbs, Quantum Mechanics and Path Integrals, McGraw-Hill Book Company, 1965.

[66] It is possible to consider alternative forms for entropy such as $k_{B} p_{m} \log \left(p_{m}-\log p_{m}\right)$. This form, however, though vanishes at $p_{m}=0$ and $p_{m}=1$ just as the entropy (8), necessitates $e^{-2 \Phi}+2 \Phi$ number of microstates, not the right amount of $1+2 \Phi$.

[67] T. Matsubara, Progr. Theoret. Phys. 14 (4) (1955) 351-378.

[68] A.A. Abrikosov, L.P. Gorkov, I.E. Dzyaloshinski, Methods of quantum field theory in statistical physics, Courier Corporation, 2012.

[69] G. Muga, R.S. Mayato, I. Egusquiza, Time in quantum mechanics, Springer Science \& Business Media, 2007.

[70] P. Busch, Found. Phys. 20 (1) (1990) 1-32.

[71] V.S. Olkhovsky, E. Recami, J. Jakiel, Phys. Rep. 398 (3) (2004) 133-178.

[72] T.E. Hartman, J. Appl. Phys. 33 (12) (1962) 3427-3433.

[73] S. Collins, D. Lowe, J. Barker, J. Phys. C: Solid State Phys. 20 (36) (1987) 6213.

[74] O. Kullie, How to understand the tunneling in attosecond experiment? ArXiv Preprint arXiv:1701.05012, 2017.

[75] D. Sokolovski, P. Hänggi, Europhys. Lett. 7 (1) (1988) 7.

[76] C. Leavens, G. Aers, Solid State Commun. 63 (12)(1987) 1101-1105.

[77] C. Leavens, G. Aers, Solid State Commun. 67 (12)(1988) 1135-1143.

[78] Transmission coefficient takes this physically correct form if Airy equation is properly solved. A detailed analysis is given by M. de Aguiar at http://sites.ifi.unicamp.br/aguiar/files/2014/10/wkb.pdf.

[79] T. Güner, Entropic Tunneling Time and its Applications, İzmir Institute of Technology, 2015.

[80] A.S. Landsman, U. Keller, J. Phys. B: At. Mol. Opt. Phys. 47 (20) (2014) 204024.

[81] J.-W. Geng, L. Qin, M. Li, W.-H. Xiong, Y. Liu, Q. Gong, L.-Y. Peng, J. Phys. B: At. Mol. Opt. Phys. 47 (20) (2014) 204027.

[82] A.K. Ghatak, E. Sauter, I.C. Goyal, Eur. J. Phys. 18 (1997) 199.

[83] A.S. Landsman, U. Keller, Phys. Rep. 547 (2015) 1-24.

[84] X. Tong, C. Lin, J. Phys. B: At. Mol. Opt. Phys. 38 (15) (2005) 2593.

[85] O. Kullie, Christian-Albrecht-University of Kiel, Germany, 1997.

[86] E. t. Clementi, D.-L. Raimondi, J. Chem. Phys. 38 (11) (1963) 2686-2689.

[87] H.B. Gray, J.R. Winkler, Proc. Natl. Acad. Sci. USA 102 (10) (2005) 3534-3539.

[88] A.A. Stuchebrukhov, Theoret. Chem. Acc. 110 (5) (2003) 291-306.

[89] J. Hupert, IRE Trans. Circuit Theory 9 (4) (1962) 425-427.

[90] J.J. Hupert, G. Ott, Amer. J. Phys. 34 (3) (1966) 260-265.

[91] T. Martin, R. Landauer, Phys. Rev. A 45 (4) (1992) 2611.

[92] R. Reddick, R. Warmack, T. Ferrell, Phys. Rev. B 39 (1) (1989) 767.

[93] T. Ferrell, J. Goundonnet, R. Reddick, S. Sharp, R. Warmack, J. Vac. Sci. Technol. B 9 (2) (1991) 525-530.

[94] N. Van Hulst, F. Segerink, F. Achten, B. Bölger, Ultramicroscopy 42 (1992) 416-421.

[95] S. Mizuno, S.-i. Tamura, Phys. Rev. B 50 (11) (1994) 7708.

[96] D. Villegas, F. de León-Pérez, R. Pérez-Alvarez, Phys. Rev. B 71 (3) (2005) 035322.

[97] D. Villegas, F. de León-Pérez, R. Pérez-Alvarez, Phys. Status Solidi B 242 (9) (2005) 1767-1770.

[98] W. Robertson, J. Ash, J. McGaugh, Amer. J. Phys. 70 (7) (2002) 689-693.

[99] I. Altfeder, A.A. Voevodin, A.K. Roy, Phys. Rev. Lett. 105 (16) (2010) 166101.

[100] H.G. Winful, Phys. Rep. 436 (1) (2006) 1-69. 